\title{
BERGMAN SPACES OF TEMPERATURE FUNCTIONS ON A CYLINDER
}

\author{
MARCOS LÓPEZ-GARCÍA
}

Received 19 April 2002

\begin{abstract}
We define the weighted Bergman space $b_{\beta}^{p}\left(S_{T}\right)$ consisting of temperature functions on the cylinder $S_{T}=S^{1} \times(0, T)$ and belonging to $L^{p}\left(\Omega_{T}, t^{\beta} d x d t\right)$, where $\Omega_{T}=(0,2) \times(0, T)$. For $\beta>-1$ we construct a family of bounded projections of $L^{p}\left(\Omega_{T}, t^{\beta} d x d t\right)$ onto $b_{\beta}^{p}\left(S_{T}\right)$. We use this to get, for $1<p<\infty$ and $1 / p+1 / p^{\prime}=1$, a duality $b_{\beta}^{p}\left(S_{T}\right)^{*}=b_{\beta^{\prime}}^{p^{\prime}}\left(S_{T}\right)$, where $\beta^{\prime}$ depends on $p$ and $\beta$.
\end{abstract}

2000 Mathematics Subject Classification: 46E22, 35K05.

1. Introduction. For $\mathbb{D}$, the open unit disk in the complex plane $\mathbb{C}$, the classic Bergman space $L_{a}^{p}$ is the subspace of holomorphic functions $f: \mathbb{D} \rightarrow \mathbb{C}$ such that $f \in L^{p}(\mathbb{D})$. It can be verified by the mean value theorem and Hölder inequality that $L_{a}^{p}$ is a closed subspace of $L^{p}(\mathbb{D})$. This implies the existence of an orthogonal projection $P$ from $L^{2}(\mathbb{D})$ onto $L_{a}^{2}$, which is called the Bergman projection. The projection $P$ can be written as an integral operator

$$
P f(z)=\int_{\mathbb{D}} K(z, w) f(w) d w
$$

for all $f \in L_{a}^{2}$, where $K(z, w)$ is the so-called Bergman reproducing kernel of $L_{a}^{2}$.

The theory of Bergman spaces has a long history. It goes back to the work of Bergman [3], who gave the first treatment of $L_{a}^{2}(\Omega)$. Today there are rich theories describing the Bergman spaces in various domains and their operators. Two of the most important classes of operators in the Bergman space theory are the Toeplitz and Hankel operators, which are defined in terms of the Bergman projection $P$. This theory was mainly developed in the late 1980 s. For a very nice exposition of the $L^{p}(\mathbb{D})$-theory of Bergman spaces, operators defined on them, and further historical references, we refer to Axler [1], Zhu [10], and a more modern approach in [2].

In this paper, we define weighted Bergman-type spaces $b_{\beta}^{p}\left(S_{T}\right)$ consisting of temperature functions on the cylinder $S_{T}=S^{1} \times(0, T)$ and belonging to $L^{p}\left(\Omega_{T}, t^{\beta} d x d t\right)$, where $\Omega_{T}=(0,2) \times(0, T)$. As in the holomorphic case, we prove that $b_{\beta}^{p}\left(S_{T}\right)$ is a Banach space. Therefore, there exist the Bergman projection and the corresponding reproducing kernel in this setting. Since the Bergman projection $P: L^{2}\left(\Omega_{T}\right) \rightarrow b^{2}\left(S_{T}\right)$ is an orthogonal projection, then it is 
bounded, but the boundedness of $P$ on $L^{p}$ is not obvious at all. We will construct a family of reproducing kernels and bounded projections $P_{\alpha}$ of $L^{p}\left(\Omega_{T}\right.$, $\left.t^{\beta} d x d t\right)$ onto $b_{\beta}^{p}\left(S_{T}\right)$, for $p>1$ and $p>(1+\beta) /(1+\alpha)$. The proof of the boundedness of these projections is based on a version of the Schur's test, and the theory of Fourier multipliers. Using the fact that $P_{\alpha}$ is bounded we obtain the duality between the Bergman spaces.

The main results of this paper are the following theorems.

THEOREM 1.1. Let $\alpha, \beta>-1$. If $p>\max (1,(1+\beta) /(1+\alpha))$, the operator $P_{\alpha}: L_{\beta}^{p}\left(\Omega_{T}\right) \rightarrow b_{\beta}^{p}\left(S_{T}\right)$ given by

$$
P_{\alpha} u(z)=\int_{\Omega_{T}} N_{\alpha}(z, w) u(w) \tau^{\alpha} d w, \quad z \in \Omega_{T},
$$

is a continuous projection onto $b_{\beta}^{p}\left(S_{T}\right)$, where

$$
N_{\alpha}(z, w)=\frac{1+\alpha}{2 T^{1+\alpha}}+\sum_{m \in \mathbb{Z}^{*}} \frac{2^{\alpha} \pi^{2(1+\alpha)} m^{2(1+\alpha)}}{\gamma\left(1+\alpha, 2 \pi^{2} m^{2} T\right)} e^{-\pi^{2} m^{2}(t+\tau)+\pi m i(x-y)} .
$$

THEOREM 1.2. Let $\alpha, \beta>-1$. If $p>\max (1,(1+\beta) /(1+\alpha))$, then $\left(b_{\beta}^{p}\left(S_{T}\right)\right)^{*}=$ $b_{(\alpha-\beta / p) p^{\prime}}^{p^{\prime}}\left(S_{T}\right)$ with respect to the duality

$$
\langle u, v\rangle_{\alpha}=\int_{\Omega_{T}} u(z) v(z) t^{\alpha} d x d t
$$

THEOREM 1.3. Let $n, d \geq 1$ and $p>1$. If $u \in b^{p}\left(S_{T}\right)$, then $t^{n / 2}\left(\partial^{n} u / \partial x^{n}\right)$, $t^{d}\left(\partial^{d} u / \partial t^{d}\right) \in L^{p}\left(\Omega_{T}\right)$. Furthermore, there are constants $C_{n}, C_{d}>0$ such that

$$
\begin{aligned}
&\left\|t^{n / 2} \frac{\partial^{n} u}{\partial x^{n}}\right\|_{L^{p}\left(\Omega_{T}\right)} \leq C_{n}\|u\|_{b^{p}\left(S_{T}\right)}, \\
&\left\|t^{d} \frac{\partial^{d} u}{\partial t^{d}}\right\|_{L^{p}\left(\Omega_{T}\right)} \leq C_{d}\|u\|_{b^{p}\left(S_{T}\right)},
\end{aligned}
$$

for all $u \in b^{p}\left(S_{T}\right)$.

The paper is organized as follows. After some preliminaries in Section 2, we define the Bergman space $b_{\beta}^{p}\left(S_{T}\right)$ in Section 3. In Section 4 , we define a family of reproducing kernels and projections $P_{\alpha}$. Finally, in Section 5 we prove the boundedness of the projections $P_{\alpha}$ and the duality between the Bergman spaces.

2. Notation and preliminary results. Throughout this paper we will use the following notation: the conjugate exponent of $p>1$ will be denoted by $p^{\prime}$, 
we will write $z=(x, t), w=(y, \tau), d z=d x d t, d w=d y d \tau, S^{1}=\left\{e^{\pi i \theta}: \theta \in\right.$ $[0,2]\}$, and $\mathbb{Z}^{*}=\{n \in \mathbb{Z}: n \neq 0\}$. For $u \in L^{1}(\mathbb{R})$, we define its Fourier transform as

$$
(\digamma u)(\varsigma)=\int_{-\infty}^{\infty} u(x) e^{-2 \pi i x \varsigma} d x
$$

For $\Omega \subset \mathbb{R}_{+}^{2}$ an open set, let

$$
H(\Omega)=\left\{u \in C^{2}(\Omega): \frac{\partial^{2} u}{\partial x^{2}}=\frac{\partial u}{\partial t} \text { on } \Omega\right\}
$$

We will call the elements of $H(\Omega)$ temperature functions. $K(x, t)$ will denote the Gauss-Weierstrass kernel. For $t>0$, let

$$
\begin{gathered}
\theta(x, t)=\sum_{n \in \mathbb{Z}} K(x+2 n, t)=\frac{1}{2} \sum_{n \in \mathbb{Z}} e^{-\pi^{2} n^{2} t+\pi n i x}, \\
\varphi(x, t)=-2 \frac{\partial \theta}{\partial x}(x, t),
\end{gathered}
$$

and for $t \leq 0$, let $K=\theta=\varphi=0$ (see [8]). Moreover,

$$
\int_{0}^{2} \theta(x, t) d x=\int_{-\infty}^{\infty} K(x, t) d x=1, \quad \forall t>0
$$

Let $Q=(0,1) \times(0,1), \Gamma=\Gamma_{1} \cup \Gamma_{2} \cup \Gamma_{3}$ (the parabolic boundary of $Q$ ), where $\Gamma_{1}=\{0\} \times[0,1), \Gamma_{2}=\{1\} \times[0,1)$, and $\Gamma_{3}=(0,1) \times\{0\}$, and let $\lambda$ be the onedimensional Lebesgue measure on $\Gamma$.

We consider the heat kernel $\tilde{K}(x, t ; \xi, \tau)$ on $Q \times \Gamma$ defined as follows:

$$
\tilde{K}(x, t ; \xi, \tau)= \begin{cases}\varphi(x, t-\tau), & \xi=0,0 \leq \tau<1, \\ \varphi(1-x, t-\tau), & \xi=1,0 \leq \tau<1, \\ \theta(x-\xi, t)-\theta(x+\xi, t), & \tau=0,0<\xi<1 .\end{cases}
$$

It is well known that if $u \in H(Q) \cap C(\bar{Q})$, then (see [4])

$$
u(x, t)=\int_{\Gamma} \tilde{K}(x, t ; \xi, \tau) u(\xi, \tau) d \lambda(\xi, \tau), \quad \forall(x, t) \in Q
$$

Conversely, if $v \in C(\Gamma)$, then

$$
u(x, t)=\int_{\Gamma} \tilde{K}(x, t ; \xi, \tau) v(\xi, \tau) d \lambda(\xi, \tau)
$$

is a temperature function on $Q$. 
REMARK 2.1. Clearly $\widetilde{K}(x, t ; \cdot) \in L^{1}(\Gamma, d \lambda)$ for all $(x, t) \in Q$. This follows from (2.7) with $v \equiv 1$.

Let $R=(a, b) \times(c, d) \subset \mathbb{R}_{+}^{2}$ be a rectangle such that $d-c=(b-a)^{2}$. If $u \in H(R) \cap C(\bar{R})$ then $u \circ \Psi \in H(Q) \cap C(\bar{Q})$, where the mapping $\Psi: Q \rightarrow R$ is defined by

$$
\Psi(\xi, \tau)=((b-a) \xi+a,(d-c) \tau+c) .
$$

By (2.6) we have

$$
u(x, t)=\int_{\Gamma_{R}} \tilde{K}\left(\Psi^{-1}(x, t) ; \Psi^{-1}(\xi, \tau)\right) u(\xi, \tau) d \lambda_{R}(\xi, \tau), \quad \forall(x, t) \in R,
$$

where $\Gamma_{R}=\Psi(\Gamma)=\Psi\left(\Gamma_{1}\right) \cup \Psi\left(\Gamma_{2}\right) \cup \Psi\left(\Gamma_{3}\right)$ and $\lambda_{R}$ is the one-dimensional Lebesgue measure normalized on each segment of $\Gamma_{R}$.

In [5] it was proved that there is a constant $C>0$ such that

$$
\theta(x, t) \leq C(1+t) K(x, t), \quad-1<x<1 .
$$

In particular for $0<t \leq T<\infty$ there is a constant $C_{T}>0$ such that

$$
\theta(x, t) \leq C_{T} K(x, t), \quad-1<x<1 .
$$

Now, suppose $f$ is a 2-periodic continuous function on $\mathbb{R}$. Then $u$ is a temperature function on $\mathbb{R}_{+}^{2}$, 2-periodic in the variable $x$, and $u(x, t) \rightarrow f(x)$ as $t \rightarrow 0$ uniformly on [0,2] if and only if (see [8, Chapter 5 , Theorem 8])

$$
u(x, t)=\int_{0}^{2} \theta(x-y, t) f(y) d y .
$$

REMARK 2.2. Since $f(x)$ is continuous on $\mathbb{R}$ and $u(x, t) \rightarrow f(x)$ uniformly on $[0,2]$, the continuity of $u$ at $t=0$ follows.

On the other hand, if

$$
u(x, t)=\int_{0}^{2} \theta(x-y, t) f(y) d y=(K(\cdot, t) * f)(x),
$$

by Minkowski's integral inequality, we have

$$
\|u(\cdot, t)\|_{L^{p}\left(S^{1}\right)} \leq\|f\|_{L^{p}\left(S^{1}\right)}, \quad \forall t>0 .
$$

The following result, proved in [6], will be useful in this paper. 
LEMMA 2.3. Let $R=(a, b) \times(c, d) \subset \mathbb{R}_{+}^{2}$ be such that $d-c=(b-a)^{2}$. If $u \in H(R) \cap C(\bar{R})$ and $\left(x_{0}, t_{0}\right)$ is the midpoint of the upper boundary of $R$, then

$$
\left|u\left(x_{0}, t_{0}\right)\right|^{p} \leq \frac{C_{p}}{|R|} \iint_{R}|u(x, t)|^{p} d x d t
$$

where $|R|$ is the area of $R$ and $C_{p}$ is a constant depending only on $p>0$.

Next, we prove a variant of Schur's lemma (see [9]). This result provides a sufficient condition for the boundedness of an integral operator $T$ defined on $L^{p}(\Omega, d \mu), 1<p<\infty$.

LEMMA 2.4. Let $1<p<\infty$. Let $(\Omega, \mu)$ be a measure space with $\mu$ a $\sigma$-finite measure, and let $N: \Omega \times \Omega \rightarrow \mathbb{C}$ and $G: \Omega \rightarrow \mathbb{R}_{+}$be measurable functions. For $a$ measurable function $f$, define

$$
T f\left(w_{1}\right)=\int_{\Omega} N\left(w_{1}, w_{2}\right) f\left(w_{2}\right) d \mu\left(w_{2}\right)
$$

Assume that there exist measurable functions $h, g: \Omega \rightarrow \mathbb{R}_{+}$and constants $a, b \geq$ 0 such that

$$
\begin{gathered}
\int_{\Omega}\left|N\left(w_{1}, w_{2}\right)\right| h\left(w_{2}\right)^{p^{\prime}} d \mu\left(w_{2}\right) \leq\left(\operatorname{ag}\left(w_{1}\right)\right)^{p^{\prime}}, \quad \mu \text {-a.e. } \\
\int_{\Omega}\left|N\left(w_{1}, w_{2}\right)\right| g\left(w_{1}\right)^{p} G\left(w_{1}\right) d \mu\left(w_{1}\right) \leq\left(b h\left(w_{2}\right)\right)^{p} G\left(w_{2}\right), \quad \mu \text {-a.e. }
\end{gathered}
$$

Then $T: L^{p}(\Omega, G d \mu) \rightarrow L^{p}(\Omega, G d \mu)$ is a bounded operator and $\|T\| \leq a b$.

Proof. By Hölder's inequality and (2.17) we have

$$
\left|T f\left(w_{1}\right)\right| \leq \operatorname{ag}\left(w_{1}\right)\left(\int_{\Omega}\left|N\left(w_{1}, w_{2}\right)\right|\left|\frac{f\left(w_{2}\right)}{h\left(w_{2}\right)}\right|^{p} d \mu\left(w_{2}\right)\right)^{1 / p}
$$

By Tonelli's theorem and (2.18) we have

$$
\int_{\Omega}\left|T f\left(w_{1}\right)\right|^{p} G\left(w_{1}\right) d \mu\left(w_{1}\right) \leq(a b)^{p} \int_{\Omega}\left|f\left(w_{2}\right)\right|^{p} G\left(w_{2}\right) d \mu\left(w_{2}\right) .
$$

That is, $\|T f\|_{L^{p}(\Omega, G d \mu)} \leq a b\|f\|_{L^{p}(\Omega, G d \mu)}$.

LEMMA 2.5. Let $\alpha, \beta>-1$ and $p>\max (1,(1+\beta) /(1+\alpha))$. For every $\delta>0$ such that

$$
\frac{\beta-\alpha}{p}<\delta<\min \left(\frac{1+\alpha}{p^{\prime}}, \frac{1+\beta}{p}\right)
$$


the function $h(t)=t^{-\delta}$ satisfies the following inequalities:

$$
\begin{aligned}
& \int_{0}^{T} \frac{\tau^{\alpha}}{(t+\tau)^{1+\alpha}} h(\tau)^{p^{\prime}} d \tau \leq C h(t)^{p^{\prime}}, \quad \forall t \in(0, T), \\
& \int_{0}^{T} \frac{\tau^{\alpha}}{(t+\tau)^{1+\alpha}} h(t)^{p} t^{\beta} d t \leq C h(\tau)^{p} \tau^{\beta}, \quad \forall \tau \in(0, T) .
\end{aligned}
$$

Proof. Let $\gamma \in \mathbb{R}$. Making the change of variable $t=\tau s$, we have

$$
\int_{0}^{T} \frac{t^{-\gamma}}{(t+\tau)^{1+\alpha}} d t \leq \tau^{-\gamma-\alpha} \int_{0}^{\infty} \frac{s^{-\gamma}}{(s+1)^{1+\alpha}} d s=C_{\alpha, \gamma} \tau^{-\gamma-\alpha}
$$

where $C_{\alpha, \gamma}=\int_{0}^{\infty}\left(s^{-\gamma} /(s+1)^{1+\alpha}\right) d s<\infty$ whenever $-\alpha<\gamma<1$. We obtain (2.22) by letting $\gamma=\delta p^{\prime}-\alpha$ and $\gamma=\delta p-\beta$, respectively.

3. The Bergman space $b_{\beta}^{p}\left(S_{T}\right)$. Let $W: \Omega \rightarrow \mathbb{R}^{+}$be a measurable function such that $W^{-p^{\prime} / p} \in L_{\text {loc }}^{1}(\Omega)$. Denote $L_{W}^{p}(\Omega)=L^{p}(\Omega, W d x d t)$ for $1 \leq p<\infty$.

Definition 3.1. We define the Bergman space $b_{W}^{p}(\Omega)$ as the subspace of temperature functions in $L_{W}^{p}(\Omega)$. That is, $b_{W}^{p}(\Omega)=H(\Omega) \cap L_{W}^{p}(\Omega)$.

We will show that $b_{W}^{p}(\Omega)$ is a closed subspace of $L_{W}^{p}(\Omega)$ and therefore a Banach space. For this aim, we will need the following result.

Proposition 3.2. Given $1 \leq p<\infty$ and $\mathscr{K} \subset \Omega$ a compact set, there is a constant $C_{\mathscr{K}}>0$ such that

$$
|u(x, t)| \leq C_{\mathscr{K}}\|u\|_{L_{W}^{p}(\Omega)},
$$

for all $(x, t) \in \mathscr{K}, u \in b_{W}^{p}(\Omega)$.

Proof. Let $\delta=d\left(\mathscr{K}, \Omega^{c}\right)>0$ and $\mathscr{K}_{0}=\{z \in \Omega: d(z, \mathscr{K}) \leq \delta / 2\}$. For every $z \in \mathscr{K}$, let $R_{z}$ be a rectangle such that $z$ is the midpoint of the upper boundary of $R_{z}$, with height $\left(R_{z}\right)=\left\{\operatorname{base}\left(R_{z}\right)\right\}^{2}$ and $R_{z} \subset B(z, \delta / 2) \subset \mathscr{K}_{0}$.

By Lemma 2.3 there is a constant $C>0$ such that

$$
\begin{aligned}
|u(z)| & \leq \frac{C}{\left|R_{z}\right|} \iint_{R_{z}}|u(y, \tau)| d y d \tau \\
& \leq \frac{C}{\left|R_{z}\right|}\|u\|_{L_{W}^{p}(\Omega)}\left(\iint_{\mathscr{K}_{0}} W^{-p^{\prime} / p} d y d \tau\right)^{1 / p^{\prime}}
\end{aligned}
$$

for all $u \in b_{W}^{p}(\Omega)$.

We conclude the proof by choosing rectangles $R_{z}$ congruent to one another, for every $z \in \mathscr{K}$. 
REMARK 3.3. If $u_{j} \rightarrow u$ in $b_{W}^{p}(\Omega)$, then $u_{j} \rightarrow u$ uniformly on each compact subset of $\Omega$.

THEOREM 3.4. For $1 \leq p<\infty, b_{W}^{p}(\Omega)$ is a closed subspace of $L_{W}^{p}(\Omega)$. Therefore $b_{W}^{p}(\Omega)$ is a Banach space.

Proof. Given $u \in L_{W}^{p}(\Omega)$, let $\left(u_{j}\right)$ be a sequence in $b_{W}^{p}(\Omega)$ such that

$$
\left\|u-u_{j}\right\|_{L_{W}^{p}(\Omega)} \longrightarrow 0
$$

We will show that $u$ is a temperature function on $\Omega$ (except on a set of measure zero).

Pick $\left(x_{0}, t_{0}\right) \in \Omega$. Let $R=(a, b) \times(c, d)$, with $d-c=(b-a)^{2}$, such that $\left(x_{0}, t_{0}\right) \in R$ and $\bar{R} \subset \Omega$. By Proposition 3.2, there is a constant $C>0$ such that

$$
\left|u_{j}(x, t)-u_{k}(x, t)\right| \leq C\left\|u_{j}-u_{k}\right\|_{L_{W}^{p}(\Omega)}, \quad \forall(x, t) \in \bar{R} .
$$

It follows that $\left(u_{j}\right)$ converges uniformly on $\bar{R}$ to a continuous function $v$.

Since $u_{j} \in H(R) \cap C(\bar{R})$, (2.9) implies that

$$
u_{j}(x, t)=\int_{\Gamma_{R}} \tilde{K}\left(\Psi^{-1}(x, t) ; \Psi^{-1}(\xi, \tau)\right) u_{j}(\xi, \tau) d \lambda_{R}(\xi, \tau), \quad \text { for }(x, t) \in R .
$$

By Remark 2.1 and the dominated convergence theorem we have

$$
\begin{aligned}
v(x, t)=\lim _{j \rightarrow \infty} u_{j}(x, t) & =\int_{\Gamma_{R}} \tilde{K}\left(\Psi^{-1}(x, t) ; \Psi^{-1}(\xi, \tau)\right) \lim _{j \rightarrow \infty} u_{j}(\xi, \tau) d \lambda_{R}(\xi, \tau) \\
& =\int_{\Gamma_{R}} \tilde{K}\left(\Psi^{-1}(x, t) ; \Psi^{-1}(\xi, \tau)\right) v(\xi, \tau) d \lambda_{R}(\xi, \tau) .
\end{aligned}
$$

Since the function $v$ is continuous on $\Gamma_{R}$ then $v$ is a temperature function on $R$. On the other hand, $u_{j} \rightarrow u$ in $L_{W}^{p}(\Omega)$ and then some subsequence of $\left(u_{j}\right)$ converges to $u$ almost everywhere on $\Omega$. Therefore $u=v$ a.e. on $R$ and $u \in H(R)$. Since $\left(x_{0}, t_{0}\right) \in \Omega$ was arbitrary, we conclude that $u \in H(\Omega)$.

We will write $b^{p}(\Omega)=b_{W}^{p}(\Omega)$ when $W \equiv 1$. Proposition 3.2 implies that the linear functional $\mathscr{F}_{z}: b^{2}(\Omega) \rightarrow \mathbb{C}$ defined by $\mathscr{F}_{z}(u)=u(z)$ is bounded for all $z \in \Omega$. Hence the Riesz representation theorem shows that there is a function $N: \Omega \times \Omega \rightarrow \mathbb{C}$ such that

$$
u(z)=\langle u, N(z, \cdot)\rangle=\int_{\Omega} u(w) N(z, w) d w
$$

for all $u \in b^{2}(\Omega), z \in \Omega$.

The function $N: \Omega \times \Omega \rightarrow \mathbb{C}$ is called the reproducing kernel of $b^{2}(\Omega)$, some of its properties are the following (see [2]):

(1) $N(z, \cdot) \in b^{2}(\Omega)$ for all $z \in \Omega$;

(2) $N$ is real-valued and symmetric; 
(3) if $\left(u_{n}\right)$ is an orthonormal basis of $b^{2}(\Omega)$, then

$$
N(z, w)=\sum_{n=1}^{\infty} u_{n}(z) \overline{u_{n}(w)} .
$$

There is a unique orthogonal projection $P: L^{2}(\Omega) \rightarrow b^{2}(\Omega)$ called the Bergman projection. We have

$$
P u(z)=\langle P u, N(z, \cdot)\rangle=\langle u, N(z, \cdot)\rangle=\int_{\Omega} u(w) N(z, w) d w .
$$

For $T>0$, we define

$$
\begin{aligned}
& C\left(\overline{S_{T}}\right)=\{u \in C(\mathbb{R} \times[0, T]): u(x, t)=u(x+2, t)\}, \\
& H\left(S_{T}\right)=\{u \in H(\mathbb{R} \times(0, T)): u(x, t)=u(x+2, t)\} .
\end{aligned}
$$

We will call the elements of $H\left(S_{T}\right)$ temperature functions on the cylinder $S_{T}$.

Definition 3.5. For $1 \leq p<\infty$, we define the Bergman space of temperature functions on the cylinder $S_{T}$ as

$$
b_{W}^{p}\left(S_{T}\right)=\left\{u \in H\left(S_{T}\right): \int_{\Omega_{T}}|u|^{p} W d z<\infty\right\},
$$

where $\Omega_{T}=(0,2) \times(0, T)$, and $W$ is a measurable function such that $W^{-p^{\prime} / p} \in$ $L_{\mathrm{loc}}^{1}\left(\Omega_{T}\right)$.

In $b_{W}^{p}\left(S_{T}\right)$ we define the following norm:

$$
\|u\|_{b_{W}^{p}\left(S_{T}\right)}=\left(\int_{\Omega_{T}}|u|^{p} W d z\right)^{1 / p} .
$$

Notice that if $u \in b_{W}^{p}\left(S_{T}\right)$, then $\left.u\right|_{\Omega_{T}} \in L_{W}^{p}\left(\Omega_{T}\right)$. Hence we write $b_{W}^{p}\left(S_{T}\right) \subset$ $L_{W}^{p}\left(\Omega_{T}\right)$.

THEOREM 3.6. For $1 \leq p<\infty, b_{W}^{p}\left(S_{T}\right)$ is a closed subspace of $L_{W}^{p}\left(\Omega_{T}\right)$. Therefore $b_{W}^{p}\left(S_{T}\right)$ is a Banach space.

Proof. Let $u \in L_{W}^{p}\left(\Omega_{T}\right)$ and let $\left(u_{j}\right)$ be a sequence in $b_{W}^{p}\left(S_{T}\right)$ such that $\left\|u-u_{j}\right\|_{L_{W}^{p}\left(\Omega_{T}\right)} \rightarrow 0$. Consider the open set $\Omega=(-1,3) \times(0, T)$ and the sequence $\left(\left.u_{j}\right|_{\Omega}\right)$. Extend the function $W: \Omega_{T} \rightarrow \mathbb{R}_{+}$to be 2-periodic in the variable $x$. Then

$$
\left\|u_{i}-u_{j}\right\|_{L_{W}^{p}(\Omega)}=2\left\|u_{i}-u_{j}\right\|_{L_{W}^{p}\left(\Omega_{T}\right)} .
$$

It follows that $\left(\left.u_{j}\right|_{\Omega}\right)$ is a Cauchy sequence in $b_{W}^{p}(\Omega)$. Since $b_{W}^{p}(\Omega)$ is a Banach space, there is $v \in b_{W}^{p}(\Omega)$ such that $\left\|v-u_{j}\right\|_{L_{W}^{p}(\Omega)} \rightarrow 0$. By Remark 3.3, the sequence $\left(\left.u_{j}\right|_{\Omega}\right)$ converges uniformly on compact subsets of $\Omega$. So,

$$
v(0, t)=\lim _{j \rightarrow \infty} u_{j}(0, t)=\lim _{j \rightarrow \infty} u_{j}(2, t)=v(2, t),
$$


for all $0<t<T$. Extend the function $v$ as a 2-periodic function in the variable $x$. This extension, which we still denote by $v$, belongs to $b_{W}^{p}\left(S_{T}\right)$ and $\| v-$ $u_{j} \|_{L_{W}^{p}\left(\Omega_{T}\right)} \rightarrow 0$. Thus $u=v$ a.e.

As in (3.7), it is shown that there is a reproducing kernel of $b^{2}\left(S_{T}\right)$, that is, a function $N: \Omega_{T} \times \Omega_{T} \rightarrow \mathbb{R}$ satisfying

$$
u(z)=\int_{\Omega_{T}} N(z, w) u(w) d w
$$

for all $u \in b^{2}\left(S_{T}\right), z \in \Omega_{T}$.

If $\left(u_{n}\right)$ is an orthonormal basis of $b^{2}\left(S_{T}\right)$, then

$$
N(z, w)=\sum_{n=1}^{\infty} u_{n}(z) \overline{u_{n}(w)}
$$

The Bergman projection $P: L^{2}\left(\Omega_{T}\right) \rightarrow b^{2}\left(S_{T}\right)$ is the integral operator given by

$$
P u(z)=\int_{\Omega_{T}} N(z, w) u(w) d w, \quad \forall z \in \Omega_{T} .
$$

We will prove that the extension of the Bergman projection $P$ to $L^{p}\left(\Omega_{T}\right)$ is bounded for all $p>1$. Actually, we will show that $P$ is bounded on certain weighted Bergman spaces.

From now on, we will be working with weights consisting of powers of the distance of a point to the base of the cylinder, that is, we consider $W_{\beta}: \mathbb{R}_{+}^{2} \rightarrow \mathbb{R}_{+}$ given by $W_{\beta}(x, t)=t^{\beta}$. Clearly $W_{\beta}^{-p^{\prime} / p} \in L_{\text {loc }}^{1}\left(\mathbb{R}_{+}^{2}\right)$.

Let $L_{\beta}^{p}\left(\Omega_{T}\right)=L_{W_{\beta}}^{p}\left(\Omega_{T}\right)$ and $b_{\beta}^{p}\left(S_{T}\right)=b_{W_{\beta}}^{p}\left(S_{T}\right)$. If $\beta>-1$, then $W_{\beta} d x d t$ is a finite measure on $\Omega_{T}$ and

$$
L_{\beta}^{p}\left(\Omega_{T}\right) \subset L_{\beta}^{1}\left(\Omega_{T}\right), \quad b_{\beta}^{p}\left(S_{T}\right) \subset b_{\beta}^{1}\left(S_{T}\right),
$$

for all $1 \leq p<\infty$.

We will show that the subspace $H\left(S_{T}\right) \cap C\left(\overline{S_{T}}\right)$ is dense in $b_{\beta}^{p}\left(S_{T}\right)$, for all $\beta>-1$. Given $u \in b_{\beta}^{p}\left(S_{T}\right)$ and $0<r<T$, we define the function $u_{r}$ as follows:

$$
u_{r}(x, t)=\int_{0}^{2} \theta(x-y, t) u(y, r) d y
$$

From (2.12) and Remark 2.2, we have that $u_{r} \in H\left(S_{T}\right) \cap C\left(\overline{S_{T}}\right)$ and $u_{r}(x, 0)=$ $u(x, r)$.

The uniqueness of the solution of the heat equation on a finite cylinder (see [8]) yields

$$
u_{r}(x, t)=u(x, t+r), \quad \text { for } 0 \leq t<T-r .
$$


As before, we have

$$
u(x, t+r)=\int_{0}^{2} \theta(x-y, r) u(y, t) d y=(K(\cdot, r) * u(\cdot, t))(x), \text { for } r>0
$$

Minkowski's integral inequality implies

$$
\int_{0}^{2}|u(x, t+r)|^{p} d x \leq \int_{0}^{2}|u(x, t)|^{p} d x, \text { for } r>0 .
$$

THEOREM 3.7. Let $\beta>-1,1 \leq p<\infty$, and $u \in b_{\beta}^{p}\left(S_{T}\right)$. Then $\lim _{r \rightarrow 0} u_{r}=u$ in $b_{\beta}^{p}\left(S_{T}\right)$.

Proof. Given $\epsilon>0$, there exists $v \in C_{C}\left(\Omega_{T}\right)$ such that $\|u-v\|_{L_{\beta}^{p}\left(\Omega_{T}\right)}<\epsilon$. We define $v_{r}: \Omega_{T} \rightarrow \mathbb{C}$ as

$$
v_{r}(x, t)= \begin{cases}v(x, t+r), & 0 \leq x \leq 2,0 \leq t \leq T-r \\ 0, & 0 \leq x \leq 2, T-r \leq t \leq T\end{cases}
$$

Clearly $v_{r} \in C\left(\overline{\Omega_{T}}\right)$. We have

$$
\left\|u-u_{r}\right\|_{b_{\beta}^{p}\left(S_{T}\right)} \leq\|u-v\|_{L_{\beta}^{p}\left(\Omega_{T}\right)}+\left\|v-v_{r}\right\|_{L_{\beta}^{p}\left(\Omega_{T}\right)}+\left\|v_{r}-u_{r}\right\|_{L_{\beta}^{p}\left(\Omega_{T}\right)} .
$$

Since $v$ is uniformly continuous on $\overline{\Omega_{T}}$, it is easy to see that $\lim _{r \rightarrow 0} \| v-$ $v_{r} \|_{L_{\beta}^{p}\left(\Omega_{T}\right)}=0$.

The uniqueness of the solution of the heat equation on a finite cylinder and (2.12) allow to write

$$
u_{r}(x, t)=\int_{0}^{2} \theta\left(x-y, t-\frac{T}{2}+r\right) u\left(y, \frac{T}{2}\right) d y, \text { for } \frac{T}{2}-r<t<T .
$$

Hence

$$
\begin{aligned}
\left\|v_{r}-u_{r}\right\|_{L_{\beta}^{p}\left(\Omega_{T}\right)}^{p} \leq & \int_{0}^{T-r} \int_{0}^{2}|v(x, t+r)-u(x, t+r)|^{p} t^{\beta} d z \\
& +\left\|u\left(\cdot, \frac{T}{2}\right)\right\|_{\infty}^{p} \int_{T-r}^{T} \int_{0}^{2}\left|\int_{0}^{2} \theta\left(x-y, t-\frac{T}{2}+r\right) d y\right|^{p} t^{\beta} d z \\
= & A_{r}+B_{r} .
\end{aligned}
$$

By (2.4) we have that $\lim _{r \rightarrow 0} B_{r}=2\|u(\cdot, T / 2)\|_{\infty}^{p} \lim _{r \rightarrow 0} \int_{T-r}^{T} t^{\beta} d t=0$.

On the other hand, by (3.22) we have

$$
\begin{aligned}
& \int_{0}^{r} \int_{0}^{2}|v(x, t+r)-u(x, t+r)|^{p} t^{\beta} d z \\
& \leq C_{p}\left\{\|v\|_{\infty}^{p} \int_{0}^{r} t^{\beta} d t+\int_{0}^{r} \int_{0}^{2}|u(x, t)|^{p} t^{\beta} d z\right\} .
\end{aligned}
$$


If $0<r \leq t$, then

$$
t^{\beta} \leq \begin{cases}(t+r)^{\beta} & \text { if } \beta \geq 0 \\ 2^{-\beta}(t+r)^{\beta} & \text { if } \beta<0 .\end{cases}
$$

So,

$$
\int_{r}^{T-r} \int_{0}^{2}|v(x, t+r)-u(x, t+r)|^{p} t^{\beta} d z \leq C_{\beta}\|v-u\|_{L_{\beta}^{p}\left(\Omega_{T}\right)}^{p}<C_{\beta} \epsilon^{p} .
$$

From (3.27) and (3.29) it follows that $\limsup _{r \rightarrow 0} A_{r} \leq C_{\beta} \epsilon^{p}$. Hence limsup $\sup _{r \rightarrow 0} \| u$ $-u_{r} \|_{L_{\beta}^{p}\left(\Omega_{T}\right)} \leq C_{\beta} \epsilon$.

Corollary 3.8. Let $\beta>-1$ and $1 \leq p<\infty$. Then $H\left(S_{T}\right) \cap C\left(\overline{S_{T}}\right)$ is dense in $b_{\beta}^{p}\left(S_{T}\right)$.

Now, to compute the reproducing kernel we need to find an orthonormal basis of $b^{2}\left(S_{T}\right)$, so if we define

$$
u_{n}(x, t)=e^{-\pi^{2} n^{2} t+\pi n i x},
$$

then $u_{n} \in b_{\beta}^{p}\left(S_{T}\right)$ for all $n \in \mathbb{Z}, \beta>-1$. We will show that $\left(u_{n}\right)$ is an orthogonal basis of $b^{2}\left(S_{T}\right)$.

LEMMA 3.9. Let $\beta>-1$ and $1 \leq p<\infty$. The linear space generated by $\left(u_{n}\right)_{n \in \mathbb{Z}}$ is dense in $b_{\beta}^{p}\left(S_{T}\right)$.

Proof. Let $u \in b_{\beta}^{p}\left(S_{T}\right)$ and $\epsilon>0$, by the previous theorem there exists $v \in C\left(\overline{S_{T}}\right) \cap H\left(S_{T}\right)$ such that

$$
\|u-v\|_{b_{\beta}^{p}\left(S_{T}\right)}<\epsilon
$$

Since the set of trigonometric polynomials is dense in $L^{p}\left(S^{1}\right)$ and $v(\cdot, 0) \in$ $C\left(S^{1}\right)$, there exists a trigonometric polynomial $q(x)=\sum_{|n| \leq N} a_{n} e^{\pi n i x}$ such that $\|v(\cdot, 0)-q\|_{L^{p}\left(S^{1}\right)}<\epsilon$.

On the other hand, by (2.12) with $f(x)=e^{\text {rnix }}$, we have

$$
u_{n}(x, t)=\int_{0}^{2} \theta(x-y, t) e^{\pi n i y} d y=\left(K(y, t) * e^{\pi n i y}\right)(x) .
$$

By (3.19) it follows that

$$
v(x, t)=\int_{0}^{2} \theta(x-y, t) v(y, 0) d y=(K(\cdot, t) * v(\cdot, 0))(x) .
$$

By (2.14) we obtain

$$
\left\|v(\cdot, t)-\sum_{|n| \leq N} a_{n} u_{n}(\cdot, t)\right\|_{L^{p}\left(S^{1}\right)}^{p} \leq\|v(\cdot, 0)-q\|_{L^{p}\left(S^{1}\right)}^{p} .
$$


Multiplying the previous inequality by $t^{\beta}$ and integrating on $(0, T)$, we get

$$
\left\|v-\sum_{|n| \leq N} a_{n} u_{n}\right\|_{b_{\beta}^{p}\left(S_{T}\right)} \leq C_{T, \beta}\|v(\cdot, 0)-q\|_{L^{p}\left(S^{1}\right)} .
$$

Therefore, $\left\|u-\sum_{|n| \leq N} a_{n} u_{n}\right\|_{b_{\beta}^{p}\left(S_{T}\right)}<C_{T, \beta}^{\prime} \epsilon$. Since $\epsilon>0$ is arbitrary the result follows.

Clearly, the sequence $\left(u_{n}\right)_{n \in \mathbb{Z}}$ defined in (3.30) is an orthogonal set in $b^{2}\left(S_{T}\right)$. It follows that the sequence $\left(\left\|u_{n}\right\|_{b^{2}\left(S_{T}\right)}^{-1} u_{n}\right)$ is an orthonormal basis of $b^{2}\left(S_{T}\right)$. We have

$$
\left\|u_{n}\right\|_{b^{2}\left(S_{T}\right)}^{2}=\frac{1}{\pi^{2} n^{2}} \gamma\left(1,2 \pi^{2} n^{2} T\right)
$$

for all $n \in \mathbb{Z}^{*}$, where $\gamma$ is defined by

$$
\gamma(\alpha, z)=\int_{0}^{z} t^{\alpha-1} e^{-t} d t \quad \text { if } \alpha, z>0
$$

Moreover, $\left\|u_{0}\right\|_{b^{2}\left(S_{T}\right)}^{2}=2 T$. By (3.16) it follows that

$$
N(z, w)=\frac{1}{2 T}+\sum_{n \in \mathbb{Z}^{*}} \frac{\pi^{2} n^{2}}{\gamma\left(1,2 \pi^{2} n^{2} T\right)} e^{-\pi^{2} n^{2}(t+\tau)+\pi n i(x-y)} .
$$

4. The projections $P_{\alpha}$. Before proving the continuity of the Bergman projection, we will study certain integral operators $P_{\alpha}$ with kernel $N_{\alpha}$. The operators $P_{\alpha}$ will turn out to be continuous projections on $b_{\beta}^{p}\left(S_{T}\right)$.

Definition 4.1. Given $\alpha>-1$, we define $N_{\alpha}: \Omega_{T} \times \Omega_{T} \rightarrow \mathbb{C}$ as

$$
N_{\alpha}(z, w)=\sum_{m \in \mathbb{Z}} c_{m, \alpha} e^{-\pi^{2} m^{2}(t+\tau)+\pi m i(x-y)},
$$

where $c_{m, \alpha}=2^{\alpha} \pi^{2(1+\alpha)} m^{2(1+\alpha)} / \gamma\left(1+\alpha, 2 \pi^{2} m^{2} T\right)$, for all $m \in \mathbb{Z}^{*}$ and $c_{0, \alpha}=$ $(1+\alpha) / 2 T^{1+\alpha}$.

Since

$$
\gamma\left(1+\alpha, 2 \pi^{2} m^{2} T\right) \geq \int_{0}^{2 \pi^{2} T} t^{\alpha} e^{-t} d t=C_{\alpha}>0,
$$

we have that $c_{m, \alpha} \leq C_{\alpha} m^{2(1+\alpha)}$, for all $m \in \mathbb{Z}^{*}$.

Using the fact that $e^{-x} \leq C_{\lambda}^{\prime} x^{-\lambda}$ for $x>0$ and $\lambda \geq 0$, we get

$$
\left|\sum_{m \in \mathbb{Z}^{*}} m^{k} e^{-\pi^{2} m^{2}(t+\tau)+\pi m i(x-y)}\right| \leq \frac{C_{k}}{t^{k / 2+1}} \sum_{m \in \mathbb{Z}^{*}} \frac{|m|^{k}}{\left(m^{2}\right)^{k / 2+1}} \leq \frac{C_{k}}{t_{0}^{k / 2+1}},
$$

for $t \geq t_{0}>0, k \geq 0$. 
Therefore, the series defining $N_{\alpha}$ converges absolutely and uniformly on $\Omega^{\prime} \times \Omega_{T}$ provided $\Omega^{\prime} \subset \Omega_{T}$ is compact, furthermore, the function $N_{\alpha}$ is bounded on $\Omega^{\prime} \times \Omega_{T}$. So $N_{\alpha} \in C^{\infty}\left(\Omega_{T} \times \Omega_{T}\right)$ and $N_{\alpha}(\cdot, w) \in H\left(S_{T}\right)$ for all $w \in \Omega_{T}$. Since $c_{m, \alpha}=c_{-m, \alpha}$, the function $N_{\alpha}$ is real valued and symmetric. If $\alpha=0, N_{\alpha}$ coincides with the reproducing kernel of $b^{2}\left(S_{T}\right)$.

DEFINITION 4.2. For $\alpha>-1, P_{\alpha}$ is the integral operator given by

$$
P_{\alpha} u(z)=\int_{\Omega_{T}} N_{\alpha}(z, w) u(w) \tau^{\alpha} d w, \quad z \in \Omega_{T}
$$

This integral is well defined for all $u \in C_{c}^{\infty}\left(\Omega_{T}\right)$. If $\alpha=0, P_{\alpha}$ is the Bergman projection.

It is easy to see that

$$
P_{\alpha}\left(e^{-\pi^{2} n^{2} t+\pi n i x}\right)=e^{-\pi^{2} n^{2} t+\pi n i x},
$$

for all $n \in \mathbb{Z}$. Therefore, $P_{\alpha}$ is a projection on the linear space generated by $\left\{e^{-\pi^{2} n^{2} t+\pi n i x}\right\}$.

We want to show the continuity of $P_{\alpha}$ on $L_{\beta}^{p}\left(\Omega_{T}\right)$. In order to do so, we analyze the following operator:

$$
T_{\alpha} u(z)=\int_{\Omega_{T}} \Theta_{\alpha}(z, w) u(w) \tau^{\alpha} d w, \quad z \in \Omega_{T},
$$

where

$$
\Theta_{\alpha}(z, w)=\theta_{\alpha}(x-y, t+\tau)=\frac{1}{2} \pi^{2(1+\alpha)} \sum_{m \in \mathbb{Z}} m^{2(1+\alpha)} e^{-\pi^{2} m^{2}(t+\tau)+\pi m i(x-y)} .
$$

The series defining $\Theta_{\alpha}$ has the same properties of convergence as $N_{\alpha}$.

REMARK 4.3. If $\alpha \in \mathbb{N}$, then $\theta_{\alpha}(x, t)=(-1)^{1+\alpha}\left(\partial^{1+\alpha} / \partial t^{1+\alpha}\right) \theta(x, t)$.

Let $K_{\alpha}(x, t)$ be the function defined as

$$
\begin{aligned}
K_{\alpha}(x, t) & =\frac{1}{2} \digamma^{-1}\left(\pi^{2(1+\alpha)} \varsigma^{2(1+\alpha)} e^{-\pi^{2} \varsigma^{2} t}\right)\left(\frac{x}{2}\right) \\
& =\frac{1}{\sqrt{\pi} t^{1+\alpha}} K(x, t) \int_{-\infty}^{\infty}\left(\sigma+i \frac{x}{2 \sqrt{t}}\right)^{2(1+\alpha)} e^{-\sigma^{2}} d \sigma
\end{aligned}
$$

where $\digamma^{-1}$ is the inverse Fourier transform with respect to the variable $\varsigma$.

We have the following estimate:

$$
\begin{aligned}
\left|K_{\alpha}(x, t)\right| & \leq \frac{C_{\alpha}}{t^{1+\alpha}} K(x, t) \int_{-\infty}^{\infty}\left(\sigma^{2(1+\alpha)}+\frac{x^{2(1+\alpha)}}{t^{1+\alpha}}\right) e^{-\sigma^{2}} d \sigma \\
& \leq \frac{C_{\alpha}}{t^{1+\alpha}} K(x, t)\left[1+\frac{x^{2(1+\alpha)}}{t^{1+\alpha}}\right] .
\end{aligned}
$$


Since $x^{\lambda} e^{-x} \leq C_{\lambda} e^{-x / 2}$ for $x, \lambda>0$, we have

$$
\begin{aligned}
\frac{x^{2(1+\alpha)}}{(4 t)^{1+\alpha}} K(x, t) & =\frac{1}{\sqrt{4 \pi t}}\left(\frac{x^{2(1+\alpha)}}{(4 t)^{1+\alpha}} e^{-x^{2} / 4 t}\right) \\
& \leq \frac{C_{\alpha}}{\sqrt{4 \pi t}} e^{-x^{2} / 8 t}=C_{\alpha} K(x, 2 t)
\end{aligned}
$$

Therefore,

$$
\left|K_{\alpha}(x, t)\right| \leq \frac{C_{\alpha}}{t^{1+\alpha}}\{K(x, t)+K(x, 2 t)\}
$$

LEMMA 4.4. For $\alpha>-1, K_{\alpha}(x, t) \in C\left(\mathbb{R}_{+}^{2}\right)$.

Proof. We write $K_{\alpha}(x, t)=\left(1 / \sqrt{\pi} t^{1+\alpha}\right) K(x, t) \psi_{\alpha}(x, t)$, where

$$
\psi_{\alpha}(x, t)=\int_{-\infty}^{\infty}\left(\sigma+i \frac{x}{2 \sqrt{t}}\right)^{2(1+\alpha)} e^{-\sigma^{2}} d \sigma
$$

The function $f(z)=z^{2(1+\alpha)}$ is analytic on $\operatorname{Im} z>0$. Also

$$
\begin{aligned}
\int_{-\infty}^{\infty} \mid(\sigma & \left.+i \frac{x}{2 \sqrt{t}}\right)^{2(1+\alpha)} \mid e^{-\sigma^{2}} d \sigma \\
\leq & \int_{-\infty}^{\infty}\left(|\sigma|+\frac{|x|}{2 \sqrt{t}}\right)^{2(1+\alpha)} e^{-\sigma^{2}} d \sigma<\infty
\end{aligned}
$$

for all $(x, t) \in \mathbb{R}_{+}^{2}$. From the dominated convergence theorem it follows that $\psi_{\alpha}(x, t) \in C\left(\mathbb{R}_{+}^{2}\right)$.

REMARK 4.5. If $\alpha \in \mathbb{N}$, then $K_{\alpha}(x, t)=(-1)^{1+\alpha}\left(\partial^{1+\alpha} / \partial t^{1+\alpha}\right) K(x, t)$.

Now we get an alternate expression for the function $\theta_{\alpha}$ in terms of the function $K_{\alpha}$.

Proposition 4.6. For $\alpha>-1, \theta_{\alpha}(x, t)=\sum_{m \in \mathbb{Z}} K_{\alpha}(x+2 m, t)$.

Proof. From (4.11) we have

$$
\left|\sum_{m \in \mathbb{Z}} K_{\alpha}(x+2 m, t)\right| \leq \frac{C_{\alpha}}{t^{1+\alpha}}\{\theta(x, t)+\theta(x, 2 t)\} .
$$

Hence, the series converges uniformly on compact subsets of $\mathbb{R}_{+}^{2}$ and therefore it is continuous. Since the series is 2-periodic in $x$, it admits a representation as a Fourier series,

$$
\sum_{m \in \mathbb{Z}} K_{\alpha}(x+2 m, t)=\sum_{m \in \mathbb{Z}} a_{m}(t) e^{\pi m i x},
$$


where convergence is in $L^{2}\left(S^{1}\right)$. Moreover,

$$
\int_{0}^{2} \sum_{m \in \mathbb{Z}}\left|K_{\alpha}(x+2 m, t)\right| d x \leq \frac{C_{\alpha}}{t^{1+\alpha}} \int_{-\infty}^{\infty}(K(x, t)+K(x, 2 t)) d x=\frac{C_{\alpha}}{t^{1+\alpha}} .
$$

By the dominated convergence theorem we have

$$
\begin{aligned}
a_{m}(t) & =\frac{1}{2} \int_{0}^{2}\left[\sum_{n \in \mathbb{Z}} K_{\alpha}(x+2 n, t)\right] e^{-\pi m i x} d x \\
& =\frac{1}{2} \sum_{n \in \mathbb{Z}} \int_{2 n}^{2 n+2} K_{\alpha}(x, t) e^{-\pi m i x} d x \\
& =\frac{1}{2} \int_{-\infty}^{\infty} K_{\alpha}(x, t) e^{-\pi m i x} d x \\
& =\digamma\left(K_{\alpha}(2 x, t)\right)(m) \\
& =\frac{1}{2} \pi^{2(1+\alpha)} m^{2(1+\alpha)} e^{-\pi^{2} m^{2} t} .
\end{aligned}
$$

The following result is the key to prove the boundedness of the operator $P_{\alpha}$. The proof is based on the Schur's test.

THEOREM 4.7. Let $\alpha, \beta>-1$. If $p>\max (1,(1+\beta) /(1+\alpha))$, the operator $T_{\alpha}: L_{\beta}^{p}\left(\Omega_{T}\right) \rightarrow b_{\beta}^{p}\left(S_{T}\right)$ given by

$$
T_{\alpha} u(z)=\int_{\Omega_{T}} \Theta_{\alpha}(z, w) u(w) \tau^{\alpha} d w, \quad z \in \Omega_{T},
$$

is bounded.

Proof. Since $\Theta_{\alpha}(\cdot, w) \in H\left(S_{T}\right)$, it follows that $T_{\alpha} u \in H\left(S_{T}\right)$ for $u \in C_{c}^{\infty}\left(\Omega_{T}\right)$. By Lemma 2.4 , it is enough to prove that for each $p$ there is a positive measurable function $h(x, t)$ such that

$$
\begin{aligned}
& \int_{\Omega_{T}}\left|\theta_{\alpha}(x-y, t+\tau)\right| h(y, \tau)^{p^{\prime}} \tau^{\alpha} d y d \tau \leq C_{\alpha} h(x, t)^{p^{\prime}}, \quad(x, t) \in \Omega_{T}, \\
& \int_{\Omega_{T}}\left|\theta_{\alpha}(x-y, t+\tau)\right| h(x, t)^{p} \tau^{\alpha} t^{\beta} d x d t \leq C_{\alpha} h(y, \tau)^{p} t^{\beta}, \quad(y, \tau) \in \Omega_{T} .
\end{aligned}
$$

By Proposition 4.6 and (4.11) we have that

$$
\left|\theta_{\alpha}(x, t)\right| \leq \frac{C_{\alpha}}{t^{1+\alpha}}(\theta(x, t)+\theta(x, 2 t)) .
$$

Using (2.11) we get for $0<x, y<2$ that

$$
\begin{aligned}
\left|\theta_{\alpha}(x-y, t+\tau)\right| \leq \frac{C_{\alpha}}{(t+\tau)^{1+\alpha}}\{ & K(x-y+2, t+\tau)+K(x-y, t+\tau) \\
& +K(x-y-2, t+\tau)+K(x-y+2,2(t+\tau)) \\
& +K(x-y, 2(t+\tau))+K(x-y-2,2(t+\tau))\} .
\end{aligned}
$$


Let $\delta>0$ be such that $(\beta-\alpha) / p<\delta<\min \left((1+\alpha) / p^{\prime},(1+\beta) / p\right)$. Set $h(x, t)=$ $t^{-\delta}$. From Lemma 2.5 we have

$$
\begin{aligned}
& \int_{0}^{T} \frac{\tau^{-\delta p^{\prime}+\alpha}}{(t+\tau)^{1+\alpha}} \int_{0}^{2} K(x-y \pm 2, \lambda(t+\tau)) d y d \tau \\
& \quad \leq \int_{0}^{T} \frac{\tau^{-\delta p^{\prime}+\alpha}}{(t+\tau)^{1+\alpha}} \int_{-\infty}^{\infty} K(y, \lambda(t+\tau)) d y d \tau \\
& \quad=\int_{0}^{T} \frac{\tau^{-\delta p^{\prime}+\alpha}}{(t+\tau)^{1+\alpha}} d \tau \\
& \quad \leq C_{\alpha} t^{-\delta p^{\prime}}
\end{aligned}
$$

From the estimate in (4.21), together with the above calculations and letting $\lambda=1,2$ we conclude that

$$
\int_{\Omega_{T}}\left|\theta_{\alpha}(x-y, t+\tau)\right| \tau^{-\delta p^{\prime}} \tau^{\alpha} d y d \tau \leq C_{\alpha} t^{-\delta p^{\prime}} .
$$

The proof of the second inequality is similar.

The theory of Fourier multipliers is another useful tool that it will help to define an isomorphism $\mu_{\alpha}$ connecting the operators $T_{\alpha}$ and $P_{\alpha}$.

DEFINITION 4.8. Given $1 \leq p \leq \infty$, a bounded sequence $\left(\mu_{n}\right)$ is a multiplier on $L^{p}\left(S^{1}\right)$ if there is $c>0$ such that

$$
\left\|\sum \mu_{n} \hat{f}(n) e^{i n x}\right\|_{L^{p}\left(S^{1}\right)} \leq c\|f\|_{L^{p}\left(S^{1}\right)}
$$

for all trigonometric polynomials $f$.

The following result about multipliers is due to Hirschman [7].

THEOREM 4.9. Let $\left(\mu_{n}\right)$ be a bounded sequence such that $\left|\mu_{n}\right|=O\left(|n|^{-\epsilon}\right)$, $0<\epsilon<1$. Then for all $p$ satisfying $(1-\epsilon) / 2<1 / p<(1+\epsilon) / 2,\left(\mu_{n}\right)$ is a multiplier on $L^{p}\left(S^{1}\right)$.

LEMMA 4.10. Let $\left(\lambda_{n}\right)$ be a bounded sequence such that $\lambda_{n}=C_{1}+O\left(|n|^{-\epsilon}\right)$ for some $C_{1} \neq 0$ and $0<C_{2} \leq\left|\lambda_{n}\right|$ for $n \in \mathbb{Z}$ and all $\epsilon>0$. Then $\left(\lambda_{n}\right)$ induces an isomorphism $\mu$ of $b_{\beta}^{p}\left(S_{T}\right)$ onto itself for all $p>1$.

Proof. By Lemma 3.9 it is enough to define the operator $\mu$ on the elements $e^{-\pi^{2} n^{2} t+\pi n i x}, n \in \mathbb{Z}:$

$$
M\left(e^{-\pi^{2} n^{2} t+\pi n i x}\right)=\lambda_{n} e^{-\pi^{2} n^{2} t+\pi n i x} .
$$

We note that $\mathcal{M}$ can be written as $\mathcal{M}=C_{1} I+\mathcal{M}^{\prime}$, where $I$ is the identity operator and $\mathcal{M}^{\prime}$ is a multiplier operator by Theorem 4.9. Thus, there exists $C>0$ such that $\|M u\|_{L^{p}\left(S^{1}\right)} \leq C\|u\|_{L^{p}\left(S^{1}\right)}$ for all trigonometric polynomials $u$. 
By hypothesis $\left(\lambda_{n}^{-1}\right)$ is a bounded sequence and $\lambda_{n}^{-1}=C_{1}^{-1}+O\left(|n|^{-\epsilon}\right)$, as before we can see that there exists a constant $c>0$ such that $c\left\|M^{-1} u\right\|_{L^{p} t\left(S^{1}\right)} \leq$ $\|u\|_{L^{p}\left(S^{1}\right)}$ for all trigonometric polynomials $u$. So,

$$
\begin{aligned}
c\left\|\sum_{|n| \leq N} a_{n} e^{-\pi^{2} n^{2} t+\pi n i x}\right\|_{L^{p}\left(S^{1}\right)}^{p} & \leq\left\|\sum_{|n| \leq N} \lambda_{n} a_{n} e^{-\pi^{2} n^{2} t+\pi n i x}\right\|_{L^{p}\left(S^{1}\right)}^{p} \\
& \leq C\left\|\sum_{|n| \leq N} a_{n} e^{-\pi^{2} n^{2} t+\pi n i x}\right\|_{L^{p}\left(S^{1}\right)}^{p} .
\end{aligned}
$$

Multiplying the inequality by $t^{\beta}$ and integrating on $(0, T)$, we have

$$
\begin{aligned}
c\left\|\sum_{|n| \leq N} a_{n} e^{-\pi^{2} n^{2} t+\pi n i x}\right\|_{b_{\beta}^{p}\left(S_{T}\right)} & \leq\left\|\sum_{|n| \leq N} \lambda_{n} a_{n} e^{-\pi^{2} n^{2} t+\pi n i x}\right\|_{b_{\beta}^{p}\left(S_{T}\right)} \\
& \leq C\left\|\sum_{|n| \leq N} a_{n} e^{-\pi^{2} n^{2} t+\pi n i x}\right\|_{b_{\beta}^{p}\left(S_{T}\right)} .
\end{aligned}
$$

5. Proof of the main theorems. By the previous work, it is easy to prove that the operator $P_{\alpha}$ is bounded on $L_{\beta}^{p}\left(\Omega_{T}\right)$.

Proof of Theorem 1.1. Since $N_{\alpha}(\cdot, w) \in H\left(S_{T}\right)$, it follows that $P_{\alpha} u \in$ $H\left(S_{T}\right)$, for $u \in C_{c}^{\infty}\left(\Omega_{T}\right)$.

Let $\lambda_{n, \alpha}=2^{-\alpha-1} \gamma\left(1+\alpha, 2 \pi^{2} n^{2} T\right)$ for every $n \in \mathbb{Z}^{*}$ and $\lambda_{0, \alpha}=2 T^{(1+\alpha)} /(1+\alpha)$. Then

$$
0<c_{\alpha}=2^{-\alpha-1} \int_{0}^{2 \pi^{2} T} t^{\alpha} e^{-t} d t \leq \lambda_{n, \alpha} \leq 2^{-\alpha-1} \int_{0}^{\infty} t^{\alpha} e^{-t} d t=C_{\alpha}
$$

for all $n \in \mathbb{Z}^{*}$. Thus, $c_{\alpha} \leq \lambda_{n, \alpha} \leq C_{\alpha}$, for all $n \in \mathbb{Z}$.

Using the fact that $e^{-t} \leq C_{\sigma}^{\prime} t^{-\sigma}$ for $t, \sigma>0$, we have that

$$
\int_{2 \pi^{2} n^{2} T}^{\infty} t^{\alpha} e^{-t} d t \leq C_{\sigma}^{\prime} \int_{2 \pi^{2} n^{2} T}^{\infty} t^{\alpha-\sigma} d t=\frac{C_{\sigma, T}}{\sigma-\alpha-1} n^{2(\alpha-\sigma+1)}
$$

provided $\alpha-\sigma<-1$. Letting $\sigma=\alpha+1+\epsilon / 2$ we have

$$
\lambda_{n, \alpha}=C_{\alpha}+O\left(|n|^{-\epsilon}\right), \quad \forall \epsilon>0 .
$$

Let $\mathcal{M}_{\alpha}$ be the isomorphism induced by the sequence $\left(\lambda_{n, \alpha}\right)$ on $b_{\beta}^{p}\left(S_{T}\right)$ (see Lemma 4.10). That is,

$$
\mu_{\alpha}\left(\sum a_{n} e^{-\pi^{2} n^{2} t+\pi n i x}\right)=\sum \lambda_{n, \alpha} a_{n} e^{-\pi^{2} n^{2} t+\pi n i x} .
$$


In particular, we have

$$
\mathcal{M}_{\alpha} N_{\alpha}(z, w)=1+\theta_{\alpha}(x-y, t+\tau)
$$

where $\mu_{\alpha}$ is acting on the variable $z$.

Since the series defining $N_{\alpha}(z, w)$ and $\Theta_{\alpha}(z, w)$ converges uniformly on the set $\{z\} \times \Omega_{T}$ we have

$$
\left(\mu_{\alpha} \circ P_{\alpha}\right) u(z)=\left(\mathbf{1}_{\alpha}+T_{\alpha}\right) u(z), \quad \forall u \in C_{c}^{\infty}\left(\Omega_{T}\right),
$$

where $\mathbf{1}_{\alpha} u(z)=\int_{\Omega_{T}} u(w) \tau^{\alpha} d w$ is a bounded operator on $L_{\beta}^{p}\left(\Omega_{T}\right)$ if $p>$ $\max (1,(1+\beta) /(1+\alpha))$. So, $P_{\alpha}$ is continuous on $L_{\beta}^{p}\left(\Omega_{T}\right)$. The result follows from (4.5) and Lemma 3.9.

COROLLARY 5.1. The Bergman projection $P: L^{p}\left(\Omega_{T}\right) \rightarrow b^{p}\left(S_{T}\right)$ is continuous for all $p>1$.

5.1. The dual space of $b_{\beta}^{p}\left(S_{T}\right)$. This section is devoted to the study of the dual space of $b_{\beta}^{p}\left(S_{T}\right)$. For $\beta>-1, p>1$, we denote by $\langle,\rangle_{\beta}$ the weighted duality between $L_{\beta}^{p}\left(\Omega_{T}\right)$, and $L_{\beta}^{p^{\prime}}\left(\Omega_{T}\right)$, that is, $\langle u, v\rangle_{\beta}=\int_{\Omega_{T}} u(z) v(z) t^{\beta} d x d t$.

Proof OF TheOrem 1.2. Let $v \in b_{(\alpha-\beta / p) p^{\prime}}^{p^{\prime}}\left(S_{T}\right)$. We define

$$
\Phi(u)=\int_{\Omega_{T}} u(z) v(z) t^{\alpha} d x d t
$$

by Hölder's inequality $\Phi \in\left(b_{\beta}^{p}\left(S_{T}\right)\right)^{*}$ and $\|\Phi\| \leq\|v\|_{b_{(\alpha-\beta / p) p^{\prime}}^{p^{\prime}}}$.

Conversely, let $\Phi \in\left(b_{\beta}^{p}\left(S_{T}\right)\right)^{*}$. Theorem 1.1 implies that $\Phi_{\alpha}=\Phi \circ P_{\alpha} \in$ $\left(L_{\beta}^{p}\left(\Omega_{T}\right)\right)^{*}$. Therefore, there is $v_{1} \in L_{\beta}^{p^{\prime}}\left(\Omega_{T}\right)$ such that

$$
\Phi_{\alpha}(u)=\left\langle u, v_{1}\right\rangle_{\beta}=\int_{\Omega_{T}} u(z) v_{1}(z) t^{\beta} d x d t
$$

for all $u \in L_{\beta}^{p}\left(\Omega_{T}\right)$.

Since $P_{\alpha}$ is a projection onto $b_{\beta}^{p}\left(S_{T}\right)$ then $\Phi(u)=\Phi_{\alpha}(u)$ for all $u \in b_{\beta}^{p}\left(\Omega_{T}\right)$. Also,

$$
\Phi_{\alpha}(u)=\Phi_{\alpha}\left(P_{\alpha} u\right)=\int_{\Omega_{T}}\left(\int_{\Omega_{T}} N_{\alpha}(z, w) u(w) \tau^{\alpha} d y d \tau\right) v_{1}(z) t^{\beta} d x d t
$$

for all $u \in L_{\beta}^{p}\left(\Omega_{T}\right)$.

Consider $u \in C_{c}^{\infty}\left(\Omega_{T}\right)$, then

$$
\begin{aligned}
\Phi_{\alpha}(u) & =\int_{\Omega_{T}}\left(\int_{\Omega_{T}} N_{\alpha}(z, w) v_{1}(z) t^{\beta} d x d t\right) u(w) \tau^{\alpha} d y d \tau \\
& =\int_{\Omega_{T}} v(w) u(w) \tau^{\alpha} d y d \tau .
\end{aligned}
$$


It was proved in Section 4 that $N_{\alpha}(z, w)$ and all of its partial derivatives are bounded on $\Omega_{T} \times \Omega^{\prime}$ with $\Omega^{\prime} \subset \Omega_{T}$ compact. Furthermore, $N_{\alpha}(z, \cdot) \in H\left(S_{T}\right)$. Since $v_{1} \in L_{\beta}^{p^{\prime}}\left(\Omega_{T}\right) \subset L_{\beta}^{1}\left(\Omega_{T}\right)$ then $v$ is a well-defined function satisfying the heat equation.

Since $C_{c}^{\infty}\left(\Omega_{T}\right)$ is dense in $L_{\beta}^{p}\left(\Omega_{T}\right)$ and $\Phi_{\alpha} \in\left(L_{\beta}^{p}\left(\Omega_{T}\right)\right)^{*}$ then $v(w) \tau^{\alpha-\beta} \in$ $L_{\beta}^{p^{\prime}}\left(\Omega_{T}\right)$, which implies that $v \in L_{(\alpha-\beta / p) p^{\prime}}^{p^{\prime}}\left(\Omega_{T}\right)$. Hence $v \in b_{(\alpha-\beta / p) p^{\prime}}^{p^{\prime}}\left(S_{T}\right)$ and it represents $\Phi$.

Finally, we need to prove that the correspondence $v \mapsto \Phi$ is injective. Since $p^{\prime}>(1 /(1+\alpha))\left(1+(\alpha-\beta / p) p^{\prime}\right)$, Theorem 1.1 implies that $P_{\alpha}$ is a continuous projection from $L_{(\alpha-\beta / p) p^{\prime}}^{p^{\prime}}\left(\Omega_{T}\right)$ onto $b_{(\alpha-\beta / p) p^{\prime}}^{p^{\prime}}\left(S_{T}\right)$.

Assume that $\Phi=0$ is represented by $v \in b_{(\alpha-\beta / p) p^{\prime}}^{p^{\prime}}\left(S_{T}\right)$. Let $u \in C_{C}^{\infty}\left(\Omega_{T}\right)$. By Fubini's theorem we have

$$
\begin{aligned}
\int_{\Omega_{T}} u(z) v(z) t^{\alpha} d x d t & =\int_{\Omega_{T}} u(z)\left(P_{\alpha} v\right)(z) t^{\alpha} d x d t \\
& =\int_{\Omega_{T}} v(w)\left(\int_{\Omega_{T}} N_{\alpha}(z, w) u(z) t^{\alpha} d x d t\right) \tau^{\alpha} d y d \tau \\
& =\int_{\Omega_{T}} v(w)\left(P_{\alpha} u\right)(w) \tau^{\alpha} d y d \tau=\Phi\left(P_{\alpha} u\right)=0 .
\end{aligned}
$$

The density of the space $C_{c}^{\infty}\left(\Omega_{T}\right)$ implies that $v=0$. By the open mapping theorem we have that the norms $\left\|\Phi_{\alpha}\right\|$ and $\|v\|_{b_{(\alpha-\beta / p) p^{\prime}}^{p^{\prime}}\left(S_{T}\right)}$ are equivalent.

Corollary 5.2. If $p>1$, then $b^{p}\left(S_{T}\right)^{*}=b^{p^{\prime}}\left(S_{T}\right)$ with the usual duality.

COROLLARY 5.3. Let $p>1$. If $p \leq(1+\beta) /(1+\alpha)$, then $P_{\alpha}: L_{\beta}^{p}\left(\Omega_{T}\right) \rightarrow b_{\beta}^{p}\left(S_{T}\right)$ is not bounded.

Proof. Note that the adjoint operator $P_{\alpha}^{*}: b_{-(\beta / p) p^{\prime}}^{p^{\prime}}\left(S_{T}\right) \rightarrow L_{-(\beta / p) p^{\prime}}^{p^{\prime}}\left(\Omega_{T}\right)$ under the usual integral pairing is given by

$$
P_{\alpha}^{*} u(z)=t^{\alpha} \int_{\Omega_{T}} N_{\alpha}(z, w) u(w) d w
$$

Since the function 1 is in $b_{-(\beta / p) p^{\prime}}^{p^{\prime}}\left(S_{T}\right)$ and $P_{\alpha}^{*} 1=c_{0, \alpha} t^{\alpha}$ is not in $L_{-(\beta / p) p^{\prime}}^{p^{\prime}}\left(\Omega_{T}\right)$ we have that $P_{\alpha}^{*}$ is unbounded. Thus, $P_{\alpha}$ is not bounded.

Finally, we give an application of the continuity of $P_{\alpha}^{*}$. We show that there is a constant $C_{n}>0$ such that $\left\|t^{n / 2}\left(\partial^{n} u / \partial x^{n}\right)\right\|_{L^{p}\left(\Omega_{T}\right)} \leq C_{n}\|u\|_{b^{p}\left(S_{T}\right)}$, for all $u \in b^{p}\left(S_{T}\right)$.

Proof of TheOrem 1.3. If $u \in b^{p}\left(S_{T}\right)$, then

$$
u(z)=\int_{\Omega_{T}} N(z, w) u(w) d w
$$


Differentiating under the integral sign leads to

$$
\frac{\partial^{n} u}{\partial x^{n}}(z)=\int_{\Omega_{T}} \frac{\partial^{n} N}{\partial x^{n}}(z, w) u(w) d w
$$

We have

$$
\frac{\partial^{n} N}{\partial x^{n}}(z, w)=\sum_{m \in \mathbb{Z}^{*}} \frac{i^{n} \pi^{2+n} m^{2+n}}{\gamma\left(1,2 \pi^{2} m^{2} T\right)} e^{-\pi^{2} m^{2}(t+\tau)+\pi m i(x-y)} .
$$

Let $D_{n}$ be the operator defined by

$$
D_{n} u(z)=\frac{\partial^{n} u}{\partial x^{n}}(z)=\int_{\Omega_{T}} \frac{\partial^{n} N}{\partial x^{n}}(z, w) u(w) d w .
$$

On the other hand, by letting $\alpha=n / 2$ in (4.1) we define the operator $T_{n}$ as follows:

$$
T_{n} u(z)=\int_{\Omega_{T}}\left(-\frac{1+n / 2}{2 T^{1+n / 2}}+N_{n / 2}(z, w)\right) u(w) d w
$$

Let $\lambda_{m, n}=2^{n / 2} \gamma\left(1,2 \pi^{2} m^{2} T\right) / i^{n} \gamma\left(1+n / 2,2 \pi^{2} m^{2} T\right)$ for every $m \in \mathbb{Z}^{*}$ and $\lambda_{0, n}=(1+n / 2) / 2 T^{1+n / 2}$. As in (5.3) we have

$$
\lambda_{m, n}=i^{n} C_{n}+i^{n} O\left(|m|^{-\epsilon}\right),
$$

for all $\epsilon>0$.

Let $\mu_{n}$ be the isomorphism induced by the sequence $\left(\lambda_{m, n}\right)$ (see Lemma 4.10). That is,

$$
\mu_{n}\left(\sum a_{m} e^{-\pi^{2} m^{2} t+\pi m i x}\right)=\sum \lambda_{m, n} a_{m} e^{-\pi^{2} m^{2} t+\pi m i x} .
$$

It is easy to see that

$$
\left(\mu_{n} \circ D_{n}\right)\left(e^{-\pi^{2} m^{2} t+\pi m i x}\right)=T_{n}\left(e^{-\pi^{2} m^{2} t+\pi m i x}\right),
$$

for all $m \in \mathbb{Z}$.

By the inequality in (4.26), we have that there is a constant $C_{n}>0$ such that

$$
\left\|D_{n} u\right\|_{L^{p}\left(S^{1}\right)}^{p}=\left\|\mathcal{M}_{n}^{-1}\left(T_{n} u\right)\right\|_{L^{p}\left(S^{1}\right)}^{p} \leq C_{n}\left\|T_{n} u\right\|_{L^{p}\left(S^{1}\right)}^{p},
$$

for all $u \in b^{p}\left(S_{T}\right)$.

By multiplying this inequality by $t^{n p / 2}$, integrating on $(0, T)$, and using Tonelli's theorem we have

$$
\left\|t^{n / 2} D_{n} u\right\|_{L^{p}\left(\Omega_{T}\right)}^{p} \leq C_{n}\left\|t^{n / 2} T_{n} u\right\|_{L^{p}\left(\Omega_{T}\right)}^{p} .
$$


From (5.12), it follows that

$$
P_{n / 2}^{*} u(z)=t^{n / 2}\left(T_{n} u\right)(z)+\frac{1+n / 2}{2 T^{1+n / 2}} t^{n / 2} \int_{\Omega_{T}} u(w) d w .
$$

By Theorem 1.1, $P_{n / 2}: L^{p}\left(\Omega_{T}\right) \rightarrow b^{p}\left(S_{T}\right)$ is a bounded projection, for all $p>1$. Hence $P_{n / 2}^{*}: b^{p}\left(S_{T}\right) \rightarrow L^{p}\left(\Omega_{T}\right)$ is a bounded operator, for all $p>1$. It follows that

$$
\begin{aligned}
\left\|t^{n / 2} D_{n} u\right\|_{L^{p}\left(\Omega_{T}\right)} & \leq C_{n}\left(\left\|P_{n / 2}^{*} u\right\|_{L^{p}\left(\Omega_{T}\right)}+\|u\|_{b^{1}\left(S_{T}\right)}\left\|t^{n / 2}\right\|_{L^{p}\left(\Omega_{T}\right)}\right) \\
& \leq C_{n}\|u\|_{b^{p}\left(S_{T}\right)} .
\end{aligned}
$$

The proof of the other inequality is similar.

ACKNOWLEDGMENTS. This work is part of my doctoral thesis at the Universidad Nacional Autónoma de México (UNAM). The thesis was supported by the Consejo Nacional de Ciencia y Tecnología (CONACyT) and the Dirección General de Estudios de Posgrado (DGEP, UNAM). I would like to thank my thesis advisor Salvador Pérez-Esteva. I am grateful to the referees for helpful suggestions.

\section{REFERENCES}

[1] S. Axler, Bergman spaces and their operators, Surveys of Some Recent Results in Operator Theory, Vol. I (J. B. Conway and B. B. Morrel, eds.), Pitman Res. Notes Math. Ser., vol. 171, Longman Sci. Tech., Harlow, 1988, pp. 1-50.

[2] S. Axler, P. Bourdon, and W. Ramey, Harmonic Function Theory, Graduate Texts in Mathematics, vol. 137, Springer-Verlag, New York, 1992.

[3] S. Bergman, The Kernel Function and Conformal Mapping, Mathematical Surveys, no. 5, American Mathematical Society, New York, 1950.

[4] J. R. Cannon, The One-Dimensional Heat Equation, Encyclopedia of Mathematics and Its Applications, vol. 23, Addison-Wesley Publishing, Massachusetts, 1984.

[5] J. R. Cannon and S. Pérez-Esteva, On the continuous dependence of the solution of a linear parabolic partial differential equation on the boundary data and the solution at an interior spatial point, Partial Differential Equations and Applications, Lecture Notes in Pure and Appl. Math., vol. 177, Marcel Dekker, New York, 1996, pp. 57-68.

[6] M. Guzmán-Partida, Hardy spaces of conjugate temperatures, Studia Math. 122 (1997), no. 2, 153-165.

[7] A. Torchinsky, Real-Variable Methods in Harmonic Analysis, Pure and Applied Mathematics, vol. 123, Academic Press, Florida, 1986.

[8] D. V. Widder, The Heat Equation, Academic Press, New York, 1975.

[9] P. Wojtaszczyk, Banach Spaces for Analysts, Cambridge Studies in Advanced Mathematics, vol. 25, Cambridge University Press, Cambridge, 1991.

[10] K. H. Zhu, Operator Theory in Function Spaces, Monographs and Textbooks in Pure and Applied Mathematics, vol. 139, Marcel Dekker, New York, 1990.

Marcos López-García: Instituto de Matemáticas, Universidad Nacional Autónoma de México, Circuito Exterior Ciudad Universitaria, México, D.F. 04510, Mexico

E-mail address: flopez@matem. unam.mx 


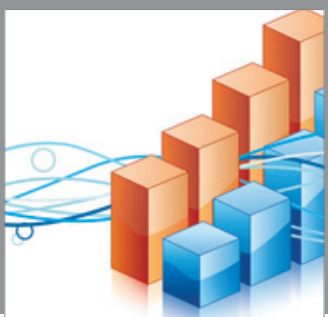

Advances in

Operations Research

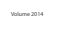

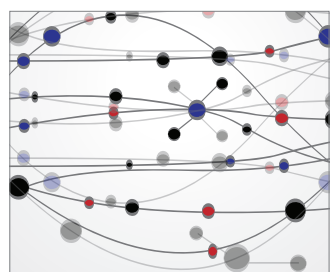

\section{The Scientific} World Journal
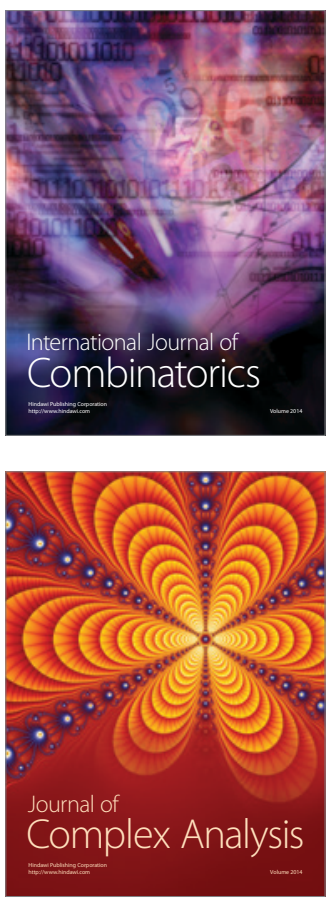

International Journal of

Mathematics and

Mathematical

Sciences
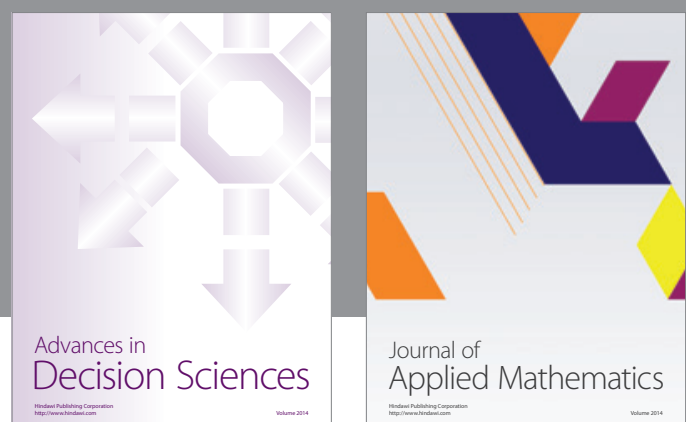

Journal of

Applied Mathematics
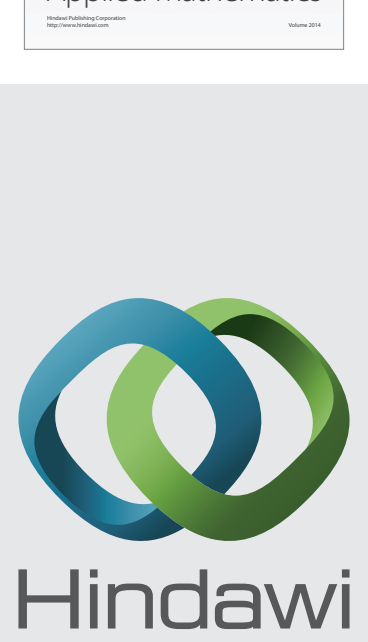

Submit your manuscripts at http://www.hindawi.com
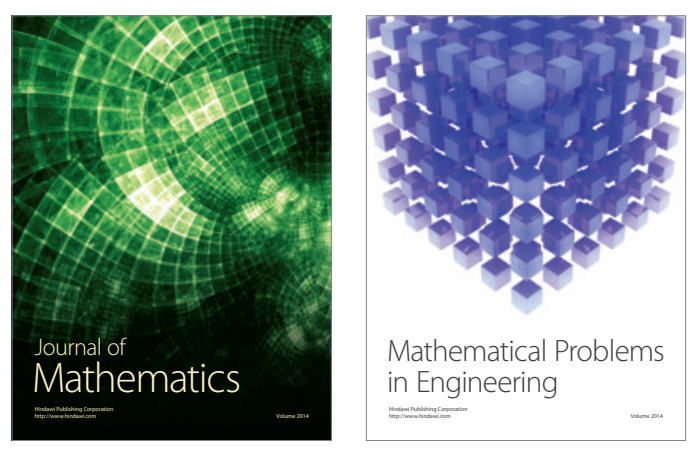

Mathematical Problems in Engineering
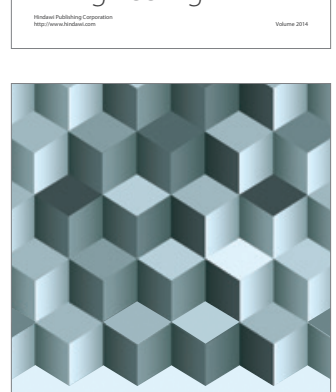

Journal of

Function Spaces
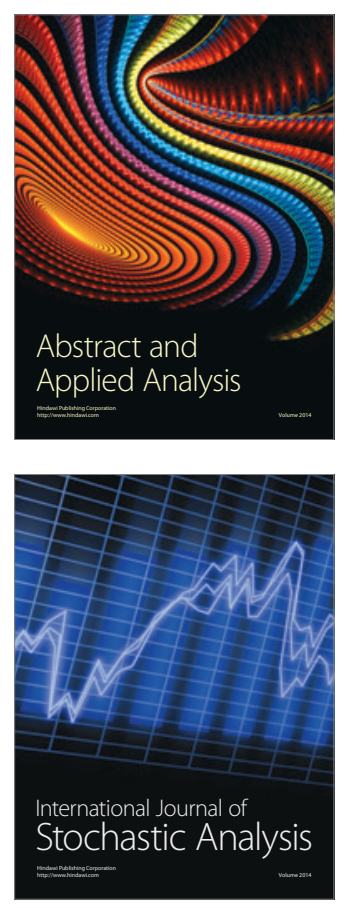

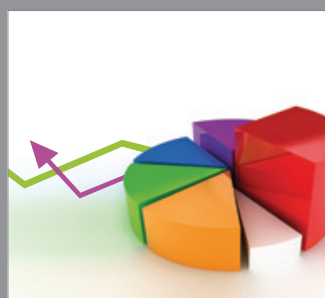

ournal of

Probability and Statistics

Promensencen
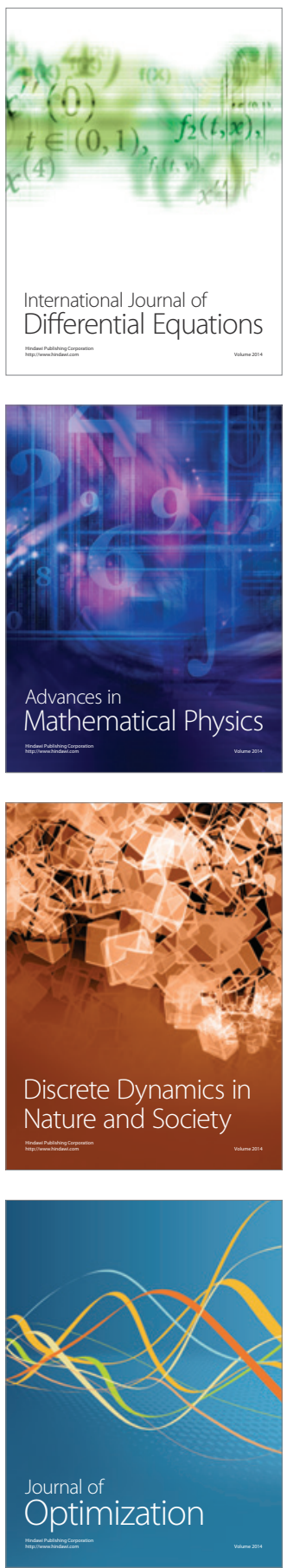